\title{
SPECTRA OF TYPE II CEPHEIDS
}

\section{T. LLOYD EVANS}

Radcliffe Observatory, South African Astronomical Observatory, South Africa

\begin{abstract}
Spectra of over 100 stars classed as type II Cepheids of short period (BL Her-type) or long period (W Vir-type) or RV Tauri stars have been obtained. A range of metallic line strength and hence presumably metal abundance is present at all periods, those having weak lines being found especially in the globular clusters and the central bulge of the Galaxy. Stars which have enhanced Carbon molecular features $\left(\mathrm{CH}, \mathrm{CN}\right.$ and sometimes $\mathrm{C}_{2}$ ) occur at all periods but are probably distributed differently in space from the other stars.
\end{abstract}

\section{DISCUSSION}

T. D. Kinman: How reliable do you believe these estimates of galactic distribution are when the samples of stars are small.

T. Lloyd Evans: The sample of $\sim 100$ stars had to be divided 9 ways, so the weight must be small The reliability of such conclusions may be estimated on completion of the program. 